\title{
Fundamental Strings, Holography, and Nonlinear Superconformal Algebras
}

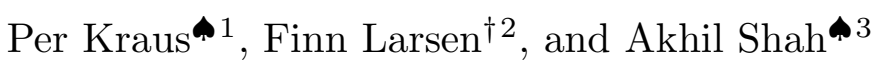 \\ - Department of Physics and Astronomy, UCLA, \\ Los Angeles, CA 90095-154\%, USA. \\ ${ }^{\dagger}$ Department of Physics and Michigan Center for Theoretical Physics, \\ University of Michigan, Ann Arbor, MI 48109-1120, USA.
}

\begin{abstract}
We discuss aspects of holography in the $A d S_{3} \times S^{p}$ near string geometry of a collection of straight fundamental heterotic strings. We use anomalies and symmetries to determine general features of the dual CFT. The symmetries suggest the appearance of nonlinear superconformal algebras, and we show how these arise in the framework of holographic renormalization methods. The nonlinear algebras imply intricate formulas for the central charge, and we show that in the bulk these correspond to an infinite series of quantum gravity corrections. We also makes some comments on the worldsheet $\sigma$-model for strings on $A d S_{3} \times S^{2}$, which is the holographic dual geometry of parallel heterotic strings in five dimensions.
\end{abstract}

August, 2007

\footnotetext{
1 pkraus@ucla.edu

2 larsenf@umich.edu

3 akhil137@ucla.edu
} 


\section{Introduction}

One can attempt to describe the fundamental string in supergravity by looking for a solution with the same symmetries and charges as a fundamental string. For example, in the classical two-derivative approximation, $N$ fundamental heterotic strings in $5 \mathrm{D}$ are described by the geometry

$$
d s_{5 E}^{2}=H^{-1 / 3}\left(-d t^{2}+d y^{2}\right)+H^{2 / 3}\left(d r^{2}+r^{2} d \Omega^{2}\right),
$$

where

$$
H=1+\frac{\sqrt{\alpha^{\prime}} N}{2 r} .
$$

Away from the origin, this solution captures the nontrivial backreacted geometry produced by the string, but at the origin the curvature diverges, signaling the need to retain higher derivative string theory corrections. The leading order corrections in heterotic string theory arise at four-derivatives $\left(R^{2}\right)$, and are known in detail [1,2, 3, 4, Keeping just corrections of this order, the fundamental string solution (given in [5.60]) then takes the form (1.1), with $H$ interpolating from the large $r$ behavior (1.2) to the $r \rightarrow 0$ near string limit

$$
H \rightarrow \frac{\ell^{3}}{r^{3}}, \quad \ell=\sqrt{\frac{\alpha^{\prime}}{2}} .
$$

The corresponding near string geometry is $A d S_{3} \times S^{2}$, a smooth space. The string coupling at the origin vanishes in the leading approximation, but the $R^{2}$-corrections stabilize it at

$$
g_{5}^{\text {het }}=2^{1 / 4} N^{-1 / 2} .
$$

The string coupling can be made arbitrarily small for large $N$, so it is meaningful to use the near string solution as the target space for a string theory $\sigma$-model. Such a description is also facilitated by the absence of Ramond sector fields. The resulting theory is interesting because, according to AdS/CFT, it provides a dual description of the original source strings. In other words, the bulk theory is the holographic dual of $N$ fundamental heterotic strings.

There are a number of obstacles to surmount in order to make this story precise. In terms of applying higher derivative corrections to the supergravity action, a general problem with any solution of order string string scale is that there is no small expansion parameter, and so one generally expects an infinite series of corrections that are all of the same order. In a similar vein, there are field redefinition ambiguities to contend with, since, for example, $g_{\mu \nu}$ and $\alpha^{\prime} R_{\mu \nu}$ are of the same order. What makes the present situation special is that symmetries and anomalies in the near horizon region are so powerful that some features of the solution are protected from corrections beyond the four-derivative 
order. An important example is that the spacetime central charges can be determined exactly in this manner, which in turn leads to precise results for black hole entropy.

The next issue appears when we consider the symmetries of the near horizon geometry of fundamental strings. If we extend our discussion to the case of a straight fundamental string in $p+3$ dimensions, then we expect the existence of an $\mathrm{AdS}_{3} \times S^{p}$ near horizon geometry. The presence of the $\mathrm{AdS}_{3}$ factor implies the existence of left and right moving Virasoro algebras [7]. Further, we expect the near horizon geometry to preserve 16 supercharges, due to the usual near horizon enhancement. Based on the worldsheet structure of the heterotic string, we thus expect a $(0,8)$ theory with $S O(p+1)$ acting as an R-symmetry. The puzzle is that in the standard list of superconformal algebras one has at most 4 right moving supercurrents.

There is at least one potential resolution of this puzzle: there exist nonlinear superconformal algebras with the required symmetries [8,9]. These algebras contain just the stress tensor, R-symmetry currents, and supercurrents, and are classified according to their finite dimensional supergroups. The algebras are nonlinear in that bilinears of the R-currents appear in the OPEs of the supercurrents. These nonlinear algebras have been studied from the algebraic perspective [10,11, 12, 13, 14, 15] but have not found many physical applications. One place where they are known to appear is as the asymptotic symmetry algebra of $\mathrm{AdS}_{3}$ supergravity based on arbitrary supergroups, as was noted in [16,17], and shown in detail in [18]. Since they naturally appear in the context of $\mathrm{AdS}_{3}$ supergravity, we are led to conjecture that the symmetry algebras of our heterotic string solutions are precisely these nonlinear superconformal algebras.

This proposal has a number of interesting implications, of which we now mention one (there are also some puzzles, as we discuss later on). The algebras are each parameterized by a parameter $k$, identified with the level of the R-symmetry current algebra. Jacobi identities give a formula for the central charge in terms of $k$ that has the structure $c(k) \sim$ $k+k^{0}+\frac{1}{k}+\ldots$. By contrast, the ordinary superconformal algebras have $c \sim k$. As we'll show explicitly, $k$ is proportional to the number of heterotic strings $N$. From (1.4) we see that the expansion in $1 / k$ is equivalent to an expansion in $g_{s}^{2}$, i.e. in Newton's constant. In other words, we find that the Jacobi identities of the nonlinear algebras imply an infinite series of quantum gravity corrections to the central charges. Using standard methods, we can translate this result into quantum gravity corrections to the entropy of black holes in this theory. We find it remarkable that (under our assumptions) these corrections are determined algebraically.

This paper is aimed at collecting some results and observations related to the holographic duals of heterotic strings, mainly based on the hypothesis that they are governed by the nonlinear superconformal algebras. We start by discussing the explicit solution for the geometry near $N$ fundamental strings in 5D when $R^{2}$-corrections are taken into 
account. For this we must dualize previous results [5],6] to the heterotic frame. We next revisit the symmetry and anomaly inflow arguments from [19,20,21] and generalize them to $A d S_{3} \times S^{p}$ solutions for all $p$. After a review of the salient aspects of the nonlinear algebras, we give results for the exact central charges including quantum corrections. For

example, we find results for the central charges for geometries like $A d S_{3} \times S^{7}$ that have not yet been found as solutions to supergravity with higher derivative terms.

In the next several sections of the paper we study the symmetry aspects of the holographic duality. We set up the holographic renormalization formalism needed to regulate and interpret infrared divergences in AdS [22,22,24,25]. We show how the nonlinear algebras follow from systematic application of AdS/CFT, reproducing the results of [18]. This classical treatment only gives the large $k$ limit of the algebra (the Poisson bracket algebra), but we explain how the full quantum algebra arises from the bulk point of view. The quantum corrrections to the central charge can be understood in the more familar context of the formula for the Sugawara central charge, and we review the corresponding $\mathrm{AdS}_{3}$ side of the story.

In the last section of the paper we make some comments on the string theory $\sigma$-model on $A d S_{3} \times S^{2}$. We review some of the standard results on string theory in $A d S_{3}$ and find that the simplest candidates reproduce most, but not all, of the features expected from the spacetime point of view. It is an interesting problem to find a workable worldsheet theory with the correct properties.

Note: As this work was being completed, a talk by Strominger at Strings 2007 reported results which overlap with some of those presented here; in particular, the relevance of the nonlinear superconformal algebras. More recently, the papers [26,27,28] have appeared, all dealing with aspects of holography for fundamental strings.

\section{Nonsingular heterotic string solutions in 5D}

In [5,6] nonsingular supergravity solutions were found representing a collection of straight heterotic strings in 5D. The supersymmetric completion of certain $R^{2}$-terms [4] were included, as they must be in order to avoid naked singularities. The explicit solutions asymptote between 5D Minkowski spacetime and a near horizon $\operatorname{AdS}_{3} \times S^{2}$ region. If we replace $\mathrm{AdS}_{3}$ by a BTZ black hole and dimensionally reduce along the horizon direction we recover the small $4 \mathrm{D}$ black holes studied in [29,30,31,32,

The solution in [5],6] is presented in terms of M-theory compactified on $K 3 \times T^{2}$, with $N$ M5-branes wrapped on $K 3$. In this description the horizon attractor value for the moduli was found to give the volume $\operatorname{Vol}\left(T^{2}\right)=M^{1}\left(2 \pi l_{P}\right)^{2}$ with the modulus $M^{1}=2^{-1 / 3} N^{2 / 3}$ 
and $l_{P}$ is the 11D Planck length. In type IIA variables 1 the corresponding string coupling is

$$
g_{s}^{I I A}=2^{-1 / 4} N^{1 / 2} .
$$

We choose to fix the total volume of $K 3 \times T^{2}$ to be the unit volume $\left(2 \pi l_{P}\right)^{6}$, so

$$
\operatorname{Vol}(K 3)=\left(M^{1}\right)^{-1}\left(2 \pi l_{P}\right)^{4}=\left(2 \pi l_{s}\right)^{4}
$$

where $l_{s}=\sqrt{\alpha^{\prime}}$.

In this paper we want to analyze physics in the heterotic string frame. For this we use six-dimensional IIA-heterotic duality, under which the dilaton transforms as $e^{\phi_{6}^{\text {het }}}=e^{-\phi_{6}^{\text {IIA }}}$, and the Einstein frame metric is invariant. To proceed, we use the standard relation between Einstein and string frame metrics,

$$
g_{\mu \nu}^{E}=e^{-\frac{4 \phi_{D}}{D-2}} g_{\mu \nu}^{S}
$$

as well as the redefinition of the dilaton under dimensional reduction,

$$
e^{-2 \phi_{D-p}}=\sqrt{\operatorname{det} g_{m n}^{S}} e^{-2 \phi_{D}},
$$

where $g_{m n}^{S}$ is the string frame metric of the compact space.

On the IIA side we find $e^{\phi_{6}^{I I A}}=e^{\phi_{10}^{I I A}}=g_{s}^{I I A}$, and therefore the heterotic string coupling is

$$
g_{s}^{\text {het }}=e^{\phi_{6}^{\text {het }}}=2^{1 / 4} N^{-1 / 2} .
$$

The heterotic string is therefore weakly coupled for large $N$.

Next, we consider the length scales of the geometry as measured in the heterotic string frame. Using the invariance of the $6 \mathrm{D}$ Einstein metric and (2.3), the conversion factor is

$$
L^{\text {het }}=\frac{1}{g_{s}^{I I A}} L^{I I A}
$$

where each length is measured with respect to the corresponding string length.

The heterotic dual is compactified to $6 \mathrm{D}$ on $T^{4}$ and there is an additional $S^{1}$ bringing the theory to $5 \mathrm{D}$. On the IIA side this circle has size $\sqrt{M^{1}}\left(2 \pi l_{P}\right)=\sqrt{M^{1}}\left(g_{s}^{I I A}\right)^{\frac{1}{3}}\left(2 \pi l_{s}\right)$. Applying the conversion factor (2.6), as well as $M^{1}=\left(g_{s}^{I I A}\right)^{\frac{4}{3}}$, we find the size on the heterotic side to be

$$
2 \pi R_{5}^{\text {het }}=2 \pi l_{s} .
$$

4 The string units are given as usual as $l_{P}=g_{s}^{1 / 3} l_{s}, R_{11}=g_{s} l_{s}=g_{s}^{2 / 3} l_{P}$. This gives $g_{s}=$ $\left(R_{11} / l_{P}\right)^{3 / 2}=\left(M^{1}\right)^{3 / 4}$. 
This is precisely the self-dual radius, which is interesting because it makes enhanced symmetry possible.

Let us now turn to the $5 \mathrm{D}$ part of the geometry. In the IIA variables the $\mathrm{AdS}_{3}$ and $S^{2}$ radii were determined to have sizes [5, 6]

$$
\ell_{S}^{I I A}=\frac{1}{2} \ell_{A}^{I I A}=\left(\frac{1}{4} N\right)^{1 / 3} l_{P}=\frac{1}{\sqrt{2}} g_{s}^{I I A} l_{s} .
$$

The heterotic geometry is also $\mathrm{AdS}_{3} \times S^{2}$ but the overall scale is different. From (2.6) we find

$$
\ell_{S}^{\text {het }}=\frac{1}{2} \ell_{A}^{\text {het }}=\frac{1}{\sqrt{2}} l_{s} .
$$

This is of string scale independent of the number of heterotic strings $N$. The string scale size of the geometry is in accord with the general scaling arguments of Sen (e.g. [33]).

One might wonder why we bothered to keep track of the precise numerical factors in the above, given that, as discussed in the introduction, the geometry is in principle subject to corrections from higher derivative terms as well as field redefinitions. The motivation for this is that our results might turn out to be unexpectedly robust. The results for the geometry were derived in the context of the supersymmetric action of [4] in which the supersymmetry variations are uncorrected by higher derivative terms. This principle removes the field redefinition ambiguity (or rather defines a preferred choice of fields). Also, we expect that the solution actually preserves 16 supersymmetries, not all of which are manifest, and it could be that some of our results are fixed by this large amount of supersymmetry.

\section{Chern-Simons terms for heterotic string solutions}

In this section we explain the significance of Chern-Simons terms for $\mathrm{AdS}_{3} \times S^{p} \times T^{7-p}$ solutions to heterotic string theory. We assume a constant dilaton and a uniform $\mathrm{H}$-flux on $\mathrm{AdS}_{3}$ carrying the charge of $N$ heterotic strings. The only explicitly known solutions of this type are the $p=2$ solutions discussed in the last section. We can nevertheless try to anticipate some features of solutions for general $p$.

Since these geometries include an $\mathrm{AdS}_{3}$ factor, there is a corresponding asymptotic symmetry algebra containing left and right moving Virasoro algebras with central charges $c_{L, R}$ [7]. Further, isometries of the $S^{p}$ yield an $S O(p+1)$ current algebra at some level $k$. We would like to relate these parameters to the number of heterotic strings $N$.

To do this we first recall that anomaly inflow [19] relates the central charges and current algebra levels to the coefficients of bulk Chern-Simons terms on $\mathrm{AdS}_{3}$ (we review 
this in section 5.) The precise relation derived in [21] lets us write the Chern-Simons couplings on $\mathrm{AdS}_{3}$ as

$$
S_{\mathrm{CS}}=\frac{c_{L}-c_{R}}{96 \pi} \int \Omega(\omega)+\frac{k}{8 \pi x_{v}} \int \Omega(A)
$$

where $\omega$ is the spin-connection, $A$ is the $S O(p+1)$ connection, and $\Omega$ is the Chern-Simons 3-form

$$
\Omega(\omega)=\operatorname{Tr}\left(\omega d \omega+\frac{2}{3} \omega^{3}\right), \quad \Omega(A)=\operatorname{Tr}_{v}\left(A d A+\frac{2}{3} A^{3}\right) .
$$

In (3.1) $x_{v}$ denotes the Dynkin index of the vector representation of $S O(p+1)$; it is equal to 2 for $S O(3)$ and 1 for the other cases.

Our strategy will be to extract terms of the form (3.1) from the complete spacetime action reduced to $\mathrm{AdS}_{3}$ in the presence of $N$ units of flux. We emphasize again that the geometries in question are of order string scale for all $N$, and so we cannot neglect $\alpha^{\prime}$ corrections when doing this. We write the bosonic sector of the heterotic string action including all higher derivative terms schematically as

$$
S=\frac{1}{2 \kappa_{10}^{2}} \int d^{10} x \sqrt{-g} \mathcal{L}\left(g_{M N}, \phi, H, A_{\text {het }}\right) .
$$

Here $\mathcal{L}$ is a function of the metric, dilaton, 3-form field strength, Yang-Mills fields, and their derivatives. In heterotic string theory the 3 -form field strength is determined by anomaly cancellation to be of the form

$$
H=d B+\frac{\alpha^{\prime}}{4} \Omega(\omega)-\frac{\alpha^{\prime}}{4} \Omega\left(A_{\text {het }}\right) .
$$

The Yang-Mills fields $A_{\text {het }}$ will be set to zero for the time being; we comment on their role at the end of the calculation.

We need an expression for the number $N$ of heterotic strings. Gauss' law for the flux $\Pi^{M N P}=\frac{\partial \mathcal{L}}{\partial H_{M N P}}$ states that the surface integral $\int^{\star} \Pi$ is independent of the radial location of the surface. In the near horizon region our ansatz gives

$$
\Pi^{M N P}=-\frac{1}{6} Q \epsilon^{M N P}
$$

where $\epsilon^{M N P}$ is the volume form of $\mathrm{AdS}_{3}$ and $Q$ is a parameter proportional to the number of fundamental strings. The solutions we consider asymptote to a flat region where all higher derivative terms are negligible. There the conserved charge can be derived from the usual two-derivative action

$$
S_{2}=\frac{1}{2 \kappa_{10}^{2}} \int d^{10} x \sqrt{-g} e^{-2 \phi}\left[R+4 \partial^{M} \phi \partial_{M} \phi-\frac{1}{12} H^{M N P} H_{M N P}\right],
$$


which gives the standard expression for $N$

$$
N=\frac{2 \pi \alpha^{\prime}}{2 \kappa_{10}^{2}} \int_{\infty}^{\star} H
$$

with the integral evaluated over the asymptotic $S^{p} \times T^{7-p}$. Flux conservation and (3.5) then relates $N$ to the near horizon data as

$$
N=\frac{2 \pi \alpha^{\prime} V_{7}}{2 \kappa_{10}^{2}} \int{ }^{\star} \Pi=\frac{2 \pi \alpha^{\prime} Q V_{7}}{2 \kappa_{10}^{2}}
$$

where $V_{7}$ is the volume of $S^{p} \times T^{7-p}$.

The 10 dimensional origin of the Chern-Simons terms on $\mathrm{AdS}_{3}$ is seen from (3.4). In particular, we are just interested in the terms in the action linear in $\Omega_{M N P}$. The relevant term is

$$
S_{C S}=\frac{1}{2 \kappa_{10}^{2}} \int d^{10} x \sqrt{-g} \frac{\alpha^{\prime}}{4} \frac{\partial \mathcal{L}}{\partial H_{M N P}} \Omega_{M N P} .
$$

We can now use (3.6), (3.8), and the definition of $\Pi$ to express this as

$$
S_{C S}=\frac{N}{8 \pi} \int_{A d S_{3}} \Omega .
$$

We have now almost achieved our goal of extracting the Chern-Simons terms (3.1) from the general action (3.3). The only missing ingredient is that so far we just considered pure $\mathrm{AdS}_{3} \times S^{p} \times T^{7-p}$. In this case the $S O(p+1)$ connection vanishes, and so in (3.10) we have $\Omega=\Omega(\omega)$. To turn on a $S O(p+1)$ connection along with the gravitational terms we replace the sphere metric $d s^{2}=\sum_{i=1}^{p+1} d y^{i} d y^{i}$ (with $\sum_{i=1}^{p+1} y^{i} y^{i}=1$ ) by $d s^{2}=$ $\sum_{i=1}^{p+1}\left(d y^{i}-A^{i j} y^{j}\right)\left(d y^{i}-A^{i k} y^{k}\right)$ (for details on this see [34]). The Chern-Simons terms then become

$$
S_{C S}=\frac{N}{8 \pi} \int_{A d S_{3}} \Omega(\omega)+\frac{N}{8 \pi} \int_{A d S_{3}} \Omega(A) .
$$

Comparing with (3.1) we finally read off the relation between the central extensions and the number of fundamental strings

$$
c_{L}-c_{R}=12 N, \quad k=\left\{\begin{array}{cc}
2 N, & p=2 \\
N, & p>2
\end{array}\right.
$$

This is the result we wanted to establish. The result is particularly useful when the level $k$ is related by supersymmetry to one of the central charges, since then the equations determine the central charges $c_{L, R}$ separately. For example, large $5 \mathrm{D}$ black strings preserve a rightmoving $\mathcal{N}=4$ algebra with $S O(3)$ R-symmetry and so $c_{R}=6 k=12 N$ [20]. However, the fundamental heterotic string solutions are expected to have more supersymmetry, and hence we can not assume this relation in general. This is the topic of the next section. 
Our derivation gives the exact answer for $c_{L}-c_{R}$ and $k$ provided that the complete contribution to the Chern-Simons terms comes from (3.4) in this manner. To establish this completely we would have to carry out a complete Kaluza-Klein reduction of the ten dimensional action down to three dimensions, which is obviously not possible given our general starting point. While it is possible that additional Chern-Simons terms are generated by the details of the Kaluza-Klein reduction, we view this as unlikely, and will henceforth assume that (3.12) are the correct expressions.

Returning to the $\Omega\left(A_{\text {het }}\right)$ term in (3.4), we can repeat the previous derivation and deduce the Chern-Simons term for the heterotic gauge fields

$$
S_{C S}=-\frac{N}{8 \pi} \int_{A d S_{3}} \Omega\left(A_{\mathrm{het}}\right) .
$$

For example, for the $S O(32)$ heterotic string, we then find a left moving $S O(32)$ current algebra at level $k=N$. For compactification on $T^{7-p}$ we also have $U(1)^{7-p} \times U(1)^{7-p}$ gauge fields. At a generic point in moduli space, these combine with the unbroken $S O(32)$ gauge fields in a $S O(23-p, 7-p)$ invariant fashion. That is to say, we find a signature $(23-p, 7-p)$ spectrum of $U(1)$ currents.

\section{Nonlinear superconformal algebras and exact central charges}

In this section we try to identify the relevant superconformal algebras governing our spacetimes. One benefit of being able to determine the correct algebra is that this will determine the quantum corrections to the central charges in (3.12).

As before, we assume the existence of $\mathrm{AdS}_{3} \times S^{p} \times T^{7-p}$ solutions and use general principles to anticipate their properties. Specifically, we expect the solution to preserve 16 supersymmetries due to the usual near horizon supersymmetry enhancement, and these should all be rightmoving supersymmetries in the boundary superconformal algebra. The global symmetry group should therefore contain one of the four supergroups with 16 supercharges:

5 Note that the relative minus sign compared to (3.11) is what tell us that this current algebra is left moving. 


\begin{tabular}{|c|c|}
\hline Supergroup & R-symmetry \\
\hline$O S p(8 \mid 2 ; R)$ & $S O(8)$ \\
\hline$F(4)$ & $\operatorname{Spin}(7)$ \\
\hline$S U(1,1 \mid 4)$ & $U(1) \times S U(4)$ \\
\hline$O S p\left(4^{*} \mid 4\right)$ & $S U(2) \times S p(4)$ \\
\hline
\end{tabular}

Table 1: Supergroups with 16 supersymmetries, and their R-symmetry groups.

We also expect the R-symmetry group to include an $S O(p+1)$ factor from isometries of the sphere and this further helps identifying the candidate group in specific examples.

We want to determine the local symmetries of the dual boundary theory for a given supergroup. We can immediately rule out the ordinary superconformal algebras, since these have at most 8 supersymmetries [35]. It is then natural to turn to the "nonlinear superconformal algebras" [8,9], since these can have additional supersymmetries with large R-symmetries, and furthermore arise as the asymptotic symmetry algebras of $\mathrm{AdS}_{3}$ supergravity based on the corresponding supergroups [18]. The nonlinearity refers to the fact that the OPE of two supercurrents includes bilinears of the R-symmetry currents. The nonlinear superconformal algebras have been classified as [8,9,11, 12, 13, 14]:

\begin{tabular}{|c|c|c|}
\hline Superalgebra & Supercurrent rep. $\rho$ & central charge \\
\hline$\widehat{O S p}(n \mid 2 ; R)$ & $\mathbf{n}$ of $S O(n)$ & $\frac{k\left(6 k+n^{2}-10\right)}{2(k+n-3)}$ \\
\hline$\widehat{S U}(1,1 \mid n)_{n \neq 2}$ & $\mathbf{n} \oplus \overline{\mathbf{n}}$ of $U(n)$ & $\frac{3 k(n+2 k)+(n-1)(1+(n+1) k)}{k+n-1}$ \\
\hline$\widehat{S U}(1,1 \mid 2) / U(1)$ & $\mathbf{2} \oplus \overline{\mathbf{2}}$ of $S U(2)$ & $6 k$ \\
\hline$\widehat{O S p}\left(4^{*} \mid 2 m\right)$ & $(\mathbf{2}, \mathbf{2 m})$ of $S U(2) \times S p(2 m)$ & $-\frac{[6 k-(2 m+1)(m-2)][k+m+2]-6 k}{k-m+2}$ \\
\hline$\widehat{D^{1}}(2,1 ; \alpha)$ & $(\mathbf{2}, \overline{\mathbf{2}})$ of $S U(2) \times S U(2)$ & $\frac{6 k_{1} k_{2}}{k_{1}+k_{2}}$ \\
\hline$\widehat{G}(3)$ & $\mathbf{7}$ of $G_{2}$ & $\frac{k(9 k+31)}{2(k+3)}$ \\
\hline$\widehat{F}(4)$ & $\mathbf{8}$ of $\operatorname{Spin}(7)$ & $\frac{2 k(2 k+11)}{k+4}$ \\
\hline
\end{tabular}

Table 2: Nonlinear superconformal algebras and their central charges.

For each entry we have recorded the Virasoro central charge which is related by Jacobi identities to the level $k$ of the affine R-symmetry algebra. Our definition of $k$ is based on 
writing the current algebra OPE as

$$
J^{a}(z) J^{b}(w) \sim \frac{k}{(z-w)^{2}} \delta^{a b}+\frac{i f^{a b c}}{z-w} J^{c}(w),
$$

in Lie algebra conventions where the Killing metric is $\delta^{a b}$ and long roots are normalized to have squared length two. We detail the normalizations in the Appendix.

Three of the cases in the above table are actually ordinary linear superconformal algebras. In particular, $\widehat{O S p}(2 \mid 2 ; R)$ is the $\mathcal{N}=2$ algebra; $\widehat{S U}(1,1 \mid 2) / U(1)$ is the small $\mathcal{N}=4$ algebra; and $\widehat{D^{1}}(2,1 ; \alpha)$ is the large $\mathcal{N}=4$ algebra.

The $\widehat{O S p}\left(4^{*} \mid 2 m\right)$ case deserves further comment. In 12 the levels of the $\widehat{S p}(2 m)$ and $\widehat{S U}(2)$ are denoted as $k_{1}$ and $k_{2}$ respectively. Jacobi identities relate them as $k_{1}=$ $-\left(k_{2}+2 m+4\right) / 2$. This relation has the important consequence that $k_{1}$ and $k_{2}$ have opposite signs for large level, clashing with unitarity. As explained in the appendix, in our conventions the $\widehat{S U}(2)$ level is given by $k=k_{2} / 2$.

For $\widehat{S U}(1,1 \mid n)_{n \neq 2} k$ refers to the level of the $\widehat{S U}(n)$ part. The level of the $\widehat{U}(1)$ (as defined in [8,9]) is fixed by Jacobi identities to be $-(k+n)$.

As noted already, these nonlinear superconformal algebras arise as the asymptotic symmetry algebras of $\mathrm{AdS}_{3}$ supergravity based on the corresponding supergroup. In particular, in [18] a classical gravity analysis was carried out yielding a nonlinear Poisson bracket algebra. The Poisson bracket algebras can be understood as the large $k$ contraction of the quantum algebras, and so the central charge that appears is given by the large $k$ limit of the formulas appearing in Table 2. The proportionality of $c$ and $k$ in the classical approximation follows directly from the fact that the classical action is proportional to $k$.

In $\mathrm{AdS}_{3}$ gravity the classical central charge is given by the Brown-Henneaux formula $c=\frac{3 \ell_{A}}{2 G_{3}}$ and so $k \sim \frac{\ell_{A}}{G_{3}} \sim \frac{\ell_{A}}{\ell_{\mathrm{Pl}}}$. The general central charge formulas $c(k)$ admit, for large $k$, an expansion in $1 / k$. We now see that these corrections are governed by powers of the Newton constant, and so represent a series of quantum gravity corrections. It is interesting that for nonlinear algebras these corrections are determined algebraically via the Jacobi identities. We explain in more detail how these come about from the gravity point of view in the following two sections.

Looking at Tables 1 and 2, we can identify evidence for and against the hypothesis that the nonlinear superconformal algebras govern the heterotic string solutions. The $\widehat{O S p}\left(4^{*} \mid 4\right)$ case has the best chance of being realized for $\mathrm{AdS}_{3} \times S^{2} \times T^{5}$, with the $S U(2)$ R-symmetry being the isometries of the $S^{2}$. It was recently shown in 28 that the $\mathrm{AdS}_{3} \times S^{2}$ solution discussed in section 2 in fact has superisometry group $O S p\left(4^{*} \mid 4\right)$, with the $S p(4)$ symmetry arising from the fermions on $T^{5}$. According to (3.12) the central charge of the supersymmetric side has the value $c_{R}=-12 N$. The magnitude of this result is desirable, although the sign is clearly not. If we instead had $c_{R}=+12 N$ then from (3.12) we would 
also deduce the desired $c_{L}=24 N$. Note that taking $N<0$ does not fix the sign problem, as this is just a parity transformation interchanging $\mathrm{L}$ and $\mathrm{R}$.

More generally, an underlying problem is that the $\widehat{S U}(2)$ and $\widehat{S p}(2 m)$ levels are forced by the Jacobi identities to have opposite signs, which means there are no unitary highest weight representations of this algebra. In particular, acting with the negative level $J_{-1}^{a}$ on a highest weight state yields a negative norm state. At the classical level, a negative level manifests itself by making the energy unbounded from below. While this is obviously cause for concern, other aspects of this proposal are sufficiently attractive to justify further study.

This same nonunitarity problem afflicts $\widehat{S U}(1,1 \mid 4)$, whose $S U(4) \cong S O(6)$ is potentially the isometry group of an $S^{5}$. There are again no unitary representation since either the $\widehat{S U}(4)$ or $\widehat{U}(1)$ levels is necessarily negative. In this case the large $N$ central charge is $c_{R}=6 N$, half of what we would expect, making the identification with the heterotic worldsheet field content obscure.

There are no obvious obstacles to having unitary representations in the $\widehat{O S p}(8 \mid 2 ; R)$ and $\widehat{F}(4)$ cases. Some evidence for unitary $\widehat{O S p}(8 \mid 2 ; R)$ representations is given in [10]. These two cases are conceivably related to $\mathrm{AdS}_{3} \times S^{7}$ and $\mathrm{AdS}_{3} \times S^{6} \times S^{1}$. The large $N$ central charges are $c_{R}=3 N$ and $c_{R}=4 N$ respectively,

\section{Quantum corrected central charges}

As we just reviewed, the nonlinear superconformal algebras imply nontrivial relations between the central charge and the current algebra level, $c_{R}(k)$. For large $k$ we can think of this in terms of an expansion in $1 / k$. From the bulk point of view this is an expansion in $g_{s}^{2}$, and so the corrections correspond to quantum corrections in the bulk. A classical computation in the bulk is only sensitive to the part of $c_{R}$ proportional to $k$.

To explain how to get the subleading terms we first consider a simpler system consisting of a gauge field on a fixed asymptotically $\mathrm{AdS}_{3}$ geometry. The action is 6

$$
S=-\frac{i k}{8 \pi} \int_{M} \operatorname{Tr}^{\prime}\left(A d A+\frac{2}{3} A^{3}\right)+\frac{k}{8 \pi} \int_{\partial M} \sqrt{g} g^{\alpha \beta} \operatorname{Tr}^{\prime}\left(A_{\alpha} A_{\beta}\right),
$$

with $A=i A^{a} T^{a}, T^{a}$ being the generators of some group $\mathcal{G}$. The notation $\operatorname{Tr}^{\prime}$ denotes a representation independent trace, $\operatorname{Tr}^{\prime}=\frac{1}{x_{\rho}} \operatorname{Tr}$, where $x_{\rho}$ is the Dynkin index. As we review momentarily, this theory possesses a level $k \mathcal{G}$-current algebra. The boundary term in (5.1) should be understood in terms of the standard holographic renormalization procedure

6 In this section we work in Euclidean signature, which accounts for the $i$ in front of the Chern-Simons term. 
applied to Chern-Simons theory [36]. This procedure also gives vanishing classical BrownHenneaux central charge, which is just a trivial consequence of the absence of dynamical gravity (we think of taking $G_{N} \rightarrow \infty$ in the Brown-Henneaux formula $c=3 \ell / 2 G_{N}$ ). On the other hand, the Sugawara stress tensor for this theory has the central charge $c=\frac{k \operatorname{dim}(\mathcal{G})}{k+g}$ where $g$ is the dual Coxeter number. This is perfectly consistent, since for large $k$ the Sugawara central charge is $\mathrm{O}(1)$, and the classical computation only sees the $\mathrm{O}(k)$ part. The nonzero central charge arises from the quantum fluctuations of the gauge fields.

We first review how to derive the current algebra from the action (5.1). The metric takes the asymptotic form

$$
d s^{2}=d \eta^{2}+e^{2 \eta / \ell} g_{\alpha \beta}^{(0)} d x^{\alpha} d x^{\beta}+\ldots
$$

We choose conformal gauge, $g_{\alpha \beta}^{(0)} d x^{\alpha} d x^{\beta}=e^{2 \omega} d w d \bar{w}$. In the gauge $A_{\eta}=0$, the boundary conditions on the components $A_{w, \bar{w}}$ require them to be finite as $\eta \rightarrow \infty$.

With the boundary term in (5.1), the variational principle corresponds to holding fixed $A_{\bar{w}}$ on the boundary but allowing $A_{w}$ to fluctuate. Indeed, the on-shell variation of the action is

$$
\delta S=\frac{k}{\pi} \int_{\partial M} d^{2} w \operatorname{Tr}^{\prime}\left(A_{w} \delta A_{\bar{w}}\right) .
$$

The corresponding current is then

$$
J_{w}^{a}=-i \pi \frac{\delta S}{\delta A_{\frac{a}{w}}^{a}}=i k A_{w}^{a}
$$

Under a gauge transformation

$$
\delta A_{\alpha}^{a}=\partial_{\alpha} \Lambda^{a}+f^{a b c} \Lambda^{b} A_{\alpha}^{c}
$$

the current varies as

$$
\delta J_{w}^{a}=i k \partial_{w} \Lambda^{a}+f^{a b c} \Lambda^{b} J_{w}^{c}
$$

We can now use standard CFT results (here in the context of classical field theory on AdS) to write

$$
\delta J_{w}^{a}\left(w_{0}\right)=i \operatorname{Res}_{w \rightarrow w_{0}} \Lambda^{b}(w) J_{w}^{b}(w) J_{w}^{a}\left(w_{0}\right)
$$

from which we read off the OPE

$$
J_{w}^{a}(w) J_{w}^{b}(0) \sim \frac{k}{w^{2}} \delta^{a b}+\frac{i f^{a b c}}{w} J^{c}(0)
$$

This confirms the existence of a level $k$ current algebra. 
Now we come the question of the central charge. This can be computed from the stress tensor two-point function on the plane, $\left\langle T_{w w}(w) T_{w w}(0)\right\rangle=\frac{c}{2 w^{4}}$. Since in (5.1) the metric only appears in the boundary term, the stress tensor is given by

$$
T_{w w}=2 \pi \frac{\delta S}{\delta g^{w w}}=k\left(A_{w}^{a}\right)^{2} .
$$

By the standard rules of AdS/CFT, the two-point function $\left\langle T_{w w}(w) T_{w w}(0)\right\rangle$ is proportional to $k^{2}\left(A_{w}^{a}(w)\right)^{2}\left(A_{w}^{a}(0)\right)^{2}$, where at the classical level $A_{w}^{a}$ is the gauge field consistent with the equations of motion and boundary conditions. In computing the stress tensor correlator on the plane we have the boundary condition $A \frac{a}{w}=0$ and hence the classical solution has $A_{w}^{a}=0$ as well. We conclude that the stress tensor correlator vanishes classically, and then so too does the classical central charge.

What makes the quantum central charge nonvanishing is that the gauge field $A_{w}^{a}$ undergoes quantum fluctuations controlled by $1 / k$. The relevant fluctuations are localized at the AdS boundary, since this is where the stress tensor lives. To quantize these fluctuations we can use the well known fact that bulk Chern-Simons theory localizes to a WZW theory on the boundary [37,38,39].

To proceed (see e.g. [40]) we write

$$
A=g^{-1} d g+g^{-1} \bar{A} g .
$$

Here $\bar{A}$ is a background connection with the prescribed boundary condition for $A_{\bar{w}}$. Substituting into (5.1) we get, in conformal gauge,

$$
\begin{aligned}
S_{\text {gauge }} & =-\frac{i k}{8 \pi} \int_{M} \operatorname{Tr}^{\prime}\left(A d A+\frac{2}{3} A^{3}\right)+\frac{k}{8 \pi} \int_{\partial M} \sqrt{g} g^{\alpha \beta} \operatorname{Tr}^{\prime}\left(A_{\alpha} A_{\beta}\right) \\
& =-\frac{i k}{8 \pi} \int_{\partial M} \operatorname{Tr}^{\prime}\left(g^{-1} \partial g g^{-1} \bar{\partial} g^{-1}-2 g^{-1} \partial g \bar{A}_{\bar{w}}\right)-\frac{i k}{24 \pi} \int_{M} \operatorname{Tr}^{\prime}\left(g^{-1} d g\right)^{3} .
\end{aligned}
$$

That is to say, we get a WZW model with the current $J \sim k g^{-1} \partial g$ coupled to the external potential $\bar{A}_{\bar{w}}$.

In this theory the conformal boundary metric $g_{\alpha \beta}^{(0)}$ couples to the Sugawara stress energy tensor, which is the quantum version of the classical formula (5.9). Computation of the $\mathrm{AdS}_{3}$ central charge from the stress tensor two-point function now becomes the same computation as in the boundary WZW model. Hence, we automotically recover the

Sugawara central charge $c=\frac{k \operatorname{dim}(\mathcal{G})}{k+g}$. The point we wish to make here is that the full result follows from a systematic application of the AdS/CFT correspondence.

\section{Nonlinear superconformal algebra from holographic renormalization}

The nonlinear superconformal algebras have a "physical" realization as the asymptotic symmetry algebras of $\mathrm{AdS}_{3}$ supergravities [16, 17, 18]. In [18] the algebras were found by 
applying the Regge-Teitelboim method [41]. In this section we will reproduce the result in the framework of holographic renormalization, which is the most convenient approach within AdS/CFT. The logic is exactly the same as led to the current algebra (5.8). In this approach, the Sugawara stress tensor contribution that was added by hand in [18] arises automatically, see (5.9).

The novelty of the nonlinear superconformal algebras lies in the OPE of two supercurrents, which has the schematic structure

$$
G(w) G(0) \sim \frac{1}{w^{3}}+\frac{J}{w^{2}}+\frac{\partial J}{w}+\frac{T}{w}+\frac{J J}{w}
$$

In particular, the nonlinearity referes to the appearance of $J J$. In the following we will derive (6.1) in the simplified case where the metric is pure $\mathrm{AdS}_{3}$. Including a general metric is completely straightforward, but clutters the computation.

The bulk supergravity action contains the usual Einstein-Hilbert term with negative cosmological constant, Chern-Simons terms for the gauge-fields, and the most relevant piece for the present purposes, the Rarita-Schwinger term for the gravitino

$$
S_{R S}=\frac{i}{16 \pi G_{N}} \int d^{3} x\left(\epsilon^{M N P} \bar{\psi}_{M}^{i} \mathcal{D}_{N}^{i j} \psi_{P}^{j}+\frac{e}{2 \ell} \bar{\psi}_{M}^{i} \Gamma^{M N} \psi_{N}^{i}\right)
$$

Here $i=1 \ldots \operatorname{dim}(\mathcal{R})$, where $\mathcal{R}$ is the representation of the gauge group under which the gravitinos transform. The covariant derivative is defined as

$$
\mathcal{D}_{M}^{i j}=\left(\partial_{M}+\frac{1}{4} \omega_{M}^{\hat{A} \hat{B}} \Gamma_{\hat{A} \hat{B}}\right) \delta^{i j}+A_{M}^{a}\left(T^{a}\right)^{i j}
$$

where $a=1, \ldots, \operatorname{dim}(\mathcal{G})$ and $T^{a}$ are generators of the gauge group $\mathcal{G}$. Three-dimensional indices are capitalized $M, N, P$, with tangent space indices being indicated with hats. Later, we will need indices on the two dimensional boundary labeled by lower-case letters $m, n$.

The OPE (6.1) will be extracted from the supersymmetry transformations of the gravitinos, which are

$$
\delta_{\epsilon} \psi_{M}^{i}=\left(\mathcal{D}_{M} \epsilon+\frac{1}{2 \ell} e_{M}^{\hat{A}} \Gamma_{\hat{A}} \epsilon\right)^{i}
$$

\subsection{Deriving the boundary supercurrent}

To derive the boundary supercurrent we must first obtain the leading radial behaviour of the gravitino from its equation of motion. As noted above, we take the metric to be pure $\mathrm{AdS}_{3}$

$$
d s^{2}=d \eta^{2}+e^{2 \eta / \ell} d w d \bar{w}
$$


We work in the gauge $\psi_{\eta}^{i}=A_{\eta}=0$, for which the equations of motion

$$
\epsilon^{M N P} \mathcal{D}_{N}^{i j} \psi_{P}^{j}+\frac{e}{2 \ell} \Gamma^{M N} \psi_{N}^{i}=0
$$

simplify to

$$
\begin{aligned}
& 0=\left(\partial_{\hat{\eta}}+\frac{1}{2 \ell} \Gamma_{\hat{\eta}}\right) \psi_{w}^{i}, \\
& 0=\left(\partial_{\hat{\eta}}+\frac{1}{2 \ell} \Gamma_{\hat{\eta}}\right) \psi_{\frac{i}{w}}^{i} .
\end{aligned}
$$

The main simplification here is that the covariant derivative in the radial $\eta$ direction has vanishing spin-connection $\mathbb{Z}$ and so becomes $\mathcal{D}_{\eta}=\partial_{\eta}=\partial_{\hat{\eta}}$. Our convention for the antisymmetric tensor is $\epsilon_{\hat{w} \hat{\bar{w}} \hat{\eta}}=+1$ and we choose $\Gamma_{\hat{A} \hat{B} \hat{C}}=-\epsilon_{\hat{A} \hat{B} \hat{C}}$. Since $\Gamma^{\hat{w} \hat{\bar{w}}}=-\Gamma^{\frac{\hat{\bar{w}}}{\hat{w}}}=$ $+\Gamma_{\hat{\eta}}$ we have $\Gamma_{\hat{\eta}}^{2}=1$, i.e., $\Gamma_{\hat{\eta}}$ is the $2 \mathrm{~d}$ chirality operator. Decomposing the spinors as

$$
\Gamma_{\hat{\eta}} \psi_{m \pm}^{i}= \pm \psi_{m \pm}^{i}
$$

we can solve (6.7) with

$$
\begin{aligned}
& \psi_{w}^{i}=\psi_{-w}^{i} e^{\eta / 2 \ell}+\psi_{+w}^{i} e^{-\eta / 2 \ell}, \\
& \psi_{\bar{w}}^{i}=\psi_{-\bar{w}}^{i} e^{\eta / 2 \ell}+\psi_{+\bar{w}}^{i} e^{-\eta / 2 \ell} .
\end{aligned}
$$

The leading boundary components $\psi_{-, w, w}^{i}$ of both spinors have negative $2 \mathrm{~d}$ chirality.

As usual in AdS/CFT, the leading boundary component plays the role of a source in the CFT coupled to a boundary current (in this case the supercurrent). To extract the current we now consider the on-shell variation of the action (6.2)

$$
\begin{aligned}
\delta S_{R S} & =\frac{i}{16 \pi G_{N}} \int d^{2} w \epsilon^{m n}\left(\bar{\psi}_{m}^{i} \delta \psi_{n}^{i}\right) \\
& =\frac{i}{16 \pi G_{N}} \int d^{2} w\left(\bar{\psi}_{w}^{i} \delta \psi_{\bar{w}}^{i}-\bar{\psi}_{\bar{w}}^{i} \delta \psi_{w}^{i}\right) \\
& =\frac{i}{16 \pi G_{N}} \int d^{2} w\left(\psi_{+w}^{i} \delta \psi_{-\bar{w}}^{i}-\psi_{-w}^{i} \delta \psi_{+\bar{w}}^{i}-\psi_{+\bar{w}}^{i} \delta \psi_{-w}^{i}+\psi_{-\bar{w}}^{i} \delta \psi_{+w}^{i}\right),
\end{aligned}
$$

where the Majorana conjugate spinor is defined by $\bar{\psi}=\psi^{T} C$ with charge conjugation matrix $C=i \sigma^{2}$.

We seek a variational principle in which we hold fixed the leading boundary components. For this to be valid we need to add a boundary term to the action to cancel the unwanted variations in $(6.10)$,

$$
\begin{aligned}
S_{b n d y} & =\frac{i}{16 \pi G_{N}} \int d^{2} x \epsilon^{m n} \psi_{+m}^{i} \psi_{-n}^{i} \\
& =\frac{i}{16 \pi G_{N}} \int d^{2} w\left(\psi_{+w}^{i} \psi_{-\bar{w}}^{i}-\psi_{+\bar{w}}^{i} \psi_{-w}^{i}\right),
\end{aligned}
$$

7 The spin-connection $\omega_{\eta}^{a b}$ only receives a contribution from gravitino induced torsion which will vanish at the boundary $\eta \rightarrow \infty$. 
leaving the desired result

$$
\delta\left(S_{R S}+S_{b n d y}\right)=\frac{i}{8 \pi G_{N}} \int d^{2} w\left(\psi_{+w}^{i} \delta \psi_{-\bar{w}}^{i}-\psi_{+\bar{w}}^{i} \delta \psi_{-w}^{i}\right) .
$$

We now define the boundary supercurrent via

$$
\delta S \equiv \frac{1}{8 \pi} \int d^{2} w \bar{G}^{i m} \delta \psi_{m}^{i},
$$

where the prefactor was fixed for later convenience. We thus find the holomorphic and anti-holomorphic boundary supercurrents

$$
G_{+w}^{i} \equiv G^{i}=\frac{i}{2 G_{N}} \psi_{+w}^{i}, \quad G_{+\bar{w}}^{i} \equiv \bar{G}^{i}=-\frac{i}{2 G_{N}} \psi_{+\bar{w}}^{i} .
$$

Although we obtain both holomorphic and antiholomorphic supercurrents, we will see that the action (6.2) only provides the boundary OPE of the holomorphic side. The corresponding antiholomorphic boundary algebra is obtained from the additional RaritaSchwinger term for a gravitino $\psi^{\tilde{i}}$ which transforms in a representation $\widetilde{\mathcal{R}}$ and with opposite sign $\mathrm{AdS}_{3}$ mass term $-\frac{1}{2 \ell} \bar{\psi}_{M}^{\tilde{i}} \Gamma^{M N} \psi^{\tilde{i}}$.

\subsection{Bulk symmetries and the boundary OPE}

The Noether theorem relates the OPE between the supercurrent $G^{i}$ and another current $\Phi$ to the variation of the supercurrent $\delta G^{i}$ under the symmetry transformation generated by $\Phi$. Here we are interested in the OPE between two supercurrents, which is then related to the supersymmetry transformation by the standard CFT expression

$$
\delta_{\epsilon} G_{w}^{i}\left(w_{0}\right)=i \operatorname{Res}_{w \rightarrow w_{0}} \epsilon^{j}(w) G_{w}^{j}(w) G_{w}^{i}\left(w_{0}\right),
$$

where the expression for $\delta_{\epsilon} G_{w}^{i}$ is that induced by the susy transformation (6.4).

According to the AdS/CFT dictionary, the two-point function on the right hand side is

$$
G^{i} G^{j}=\left.\frac{\delta^{2} S}{\delta \psi_{-\bar{w}}^{i} \delta \psi_{-\bar{w}}^{j}}\right|_{\psi_{-w}^{j}=\psi_{-\bar{w}}^{j}=0}
$$

The boundary conditions $\psi_{-w}^{i}=\psi_{-\bar{w}}^{i}=0$ ensure that the external sources are turned off.

Our susy transformations should preserve our gauge choice $\psi_{\eta}^{i}=0$, so we need

$$
\delta_{\epsilon} \psi_{\eta}^{i} \equiv 0=\left(\partial_{\hat{\eta}}+\frac{1}{2 \ell} \Gamma_{\hat{\eta}}\right) \epsilon^{i},
$$


which determines the radial dependence of the spinorial parameter as

$$
\epsilon^{i}=\epsilon_{-}^{i} e^{\eta / 2 \ell}+\epsilon_{+}^{i} e^{-\eta / 2 \ell}
$$

by repeating the manipulations yielding (6.7)-(6.9).

A symmetry transformation should also be one that leaves the sources invariant, so we need $\delta \psi_{-, w, \bar{w}}^{i}=0$. This condition relates $\epsilon_{+}^{i}$ and $\epsilon_{-}^{i}$ as follows. We need the covariant derivatives

$$
\mathcal{D}_{w}+\frac{1}{2 \ell} e_{w}^{\hat{A}} \Gamma_{\hat{A}}=\partial_{w}+A_{w}+\frac{1}{\ell} e_{w}^{\hat{w}} \Gamma_{\hat{w}}, \quad \mathcal{D}_{\bar{w}}+\frac{1}{2 \ell} e \frac{\hat{A}}{w} \Gamma_{\hat{A}}=\partial_{\bar{w}}+A_{\bar{w}},
$$

and a representation of the Gamma matrices satisfying our conventions

$$
\Gamma_{\hat{\eta}}=\left(\begin{array}{cc}
1 & 0 \\
0 & -1
\end{array}\right), \quad \Gamma_{\hat{w}}=\left(\begin{array}{cc}
0 & 0 \\
-\sqrt{2} & 0
\end{array}\right), \quad \Gamma_{\hat{\bar{w}}}=\left(\begin{array}{cc}
0 & -\sqrt{2} \\
0 & 0
\end{array}\right) .
$$

Then the remaining bulk supersymmetry transformations read

$$
\begin{aligned}
& \delta \psi_{w}^{i}=\left[\left(\partial_{w}+A_{w}\right)\left(\begin{array}{ll}
1 & 0 \\
0 & 1
\end{array}\right)+\frac{1}{\ell \sqrt{2}}\left(\begin{array}{cc}
0 & 0 \\
-\sqrt{2} & 0
\end{array}\right)\right]_{j}^{i} \epsilon^{j}, \\
& \delta \psi \frac{i}{w}=\left[\left(\partial_{\bar{w}}+A_{\bar{w}}\right)\left(\begin{array}{ll}
1 & 0 \\
0 & 1
\end{array}\right)\right]_{j}^{i} \epsilon^{j} .
\end{aligned}
$$

The constraint $\delta \psi_{-w}^{i}=0$ now determines $\epsilon_{+}$as

$$
\epsilon_{+}^{i}=\ell\left(\partial_{w}+A_{w}\right)_{j}^{i} \epsilon_{-}^{j}
$$

and $\delta \psi_{-\bar{w}}^{i}=0$ requires

$$
\left(\partial_{\bar{w}}+A_{\bar{w}}\right)_{j}^{i} \epsilon_{-}^{j}=0
$$

From (6.23) we find that the supersymmetry parameter is covariantly holomorphic, as expected for the holomorphic part of the boundary algebra. Collecting results, we find the variation of the supercurrent

$$
\begin{aligned}
\delta G^{i} & =\frac{i}{2 G_{N}} \delta \psi_{+w}^{i} \\
& =\frac{i}{2 G_{N}}\left(\partial_{w}+A_{w}\right)_{j}^{i} \epsilon_{+}^{j} \\
& =\frac{i \ell}{2 G_{N}}\left(\partial_{w}+A_{w}\right)_{j}^{i}\left(\partial_{w}+A_{w}\right)_{k}^{j} \epsilon_{-}^{k} .
\end{aligned}
$$

We can now read off the boundary OPE of two supercurrents by using (6.15) and the expression (5.4) for the boundary current $J_{w}^{a}=i k A_{w}^{a}$. We find

$$
G^{i}(w) G^{j}(0) \sim \frac{\ell}{G_{N}}\left(\frac{\delta^{i j}}{w^{3}}+\frac{1}{k} \frac{J^{a}\left(T^{a}\right)^{i j}}{w^{2}}+\frac{1}{2 k} \frac{\partial_{w} J_{w}^{a}\left(T^{a}\right)^{i j}}{w}+\frac{1}{2 k^{2}} \frac{J_{w}^{a} J_{w}^{b}\left(T^{a}\right)^{i k}\left(T^{b}\right)_{k}^{j}}{w}\right) .
$$


To compare with the CFT literature we need to trade $\ell$ for $k$. We first use the BrownHenneaux formula $c=3 \ell / 2 G_{N}$. For the simple supergroups with irreducible $\rho$ (the cases $O S p(m \mid 2 ; R), G(3)$ and $F(4))$ the large $k$ relation between $c$ and $k$ is 8

$$
c=\frac{3 k}{2 \chi}, \quad \chi=\frac{x_{\rho} \operatorname{dim}(\mathcal{G})}{\operatorname{dim}(\rho)(\operatorname{dim}(\rho)-1)},
$$

as can be verified from Table 2 . This gives

$$
G^{i}(w) G^{j}(0) \sim \frac{k}{\chi} \frac{\delta^{i j}}{w^{3}}+\frac{1}{\chi} \frac{J^{a}\left(T^{a}\right)^{i j}}{w^{2}}+\frac{1}{2 \chi} \frac{\partial_{w} J_{w}^{a}\left(T^{a}\right)^{i j}}{w}+\frac{1}{2 \chi k} \frac{J_{w}^{a} J_{w}^{b}\left(T^{a}\right)^{i k}\left(T^{b}\right)_{k}^{j}}{w} .
$$

The term quadratic in the currents includes the Sugawara contribution to the stress-tensor (see (5.9)) so we can write the complete expansion as

$$
G^{i}(w) G^{j}(0) \sim \frac{k}{\chi} \frac{\delta^{i j}}{w^{3}}+\frac{1}{\chi} \frac{J^{a}\left(T^{a}\right)^{i j}}{w^{2}}+\frac{2 T \delta^{i j}}{w}+\frac{1}{2 \chi} \frac{\partial_{w} J_{w}^{a} T^{a}}{w}+\frac{1}{2 \chi k} \frac{J_{w}^{a} J_{w}^{b} P_{a b}^{i j}}{w},
$$

where $P_{a b}^{i j}=\frac{1}{2}\left\{T^{a}, T^{b}\right\}^{i j}-2 \chi \delta_{a b} \delta^{i j}$. This is our final result for the OPE of two supercurrents. For specific groups the Lie algebra may be such that $P_{a b}^{i j}=0$ identically, and so the nonlinear term in (6.28) vanishes. This happens for $S O(3)$, corresponding to the usual $\mathcal{N}=4 \mathrm{SCA}$. But for more general groups the nonlinearity persists. The coefficients of all the terms in (6.28) agree for large $k$ with those previously determined by analyzing the Jacobi identities of the quantum nonlinear SCA (see e.g., [15].)

As we have emphasized, the computation done here is classical and so our expressions are valid only up to corrections suppressed by $\mathcal{O}(1 / k)$. For instance, in the quantum treatment the $J J$ product in (6.27) requires normal ordering. On the one hand, the $1 / k$ corrections can be determined algebraically by demanding a consistent operator algebra obeying the Jacobi identities. On the other hand, we can also understand the origin of the $1 / k$ corrections from the gravity point of view, in the same spirit as discussed at the end of section 5. The corrections come from quantizing the fluctuations around AdS, and in particular the pure gauge modes localized near the boundary. In section 5 these modes were described by a WZW model, while in the present case they are described by a super Liouville theory, as shown in [18]. Quantizing this theory will then yield the full central charge expressions of Table 2 .

\section{String Theory on $\mathbf{A d S}_{3} \times S^{2}$}

In this section we make some comments on the heterotic $\sigma$-model with $\mathrm{AdS}_{3} \times S^{2}$ target space. This is the holographic dual of $N$ fundamental heterotic strings.

\footnotetext{
8 There are analogous case-by-case formulas for the other groups.
} 
Let us begin by recalling the basics of heterotic string theory on a $S L(2)_{k} \times S U(2)_{k^{\prime}} \times$ $U(1)^{4}$ target space. This is just a sum of $W Z W$-models. Keeping track of just the bosons, the world-sheet central charge becomes

$$
c_{B, \mathrm{ws}}=\frac{3 k_{B}}{k_{B}+2}+\frac{3 k_{B}^{\prime}}{k_{B}^{\prime}-2}+4 .
$$

To get the critical central charge we need $c_{B \text {,ws }}=10$, as for 10 free bosons. This gives the condition

$$
k_{B}=k_{B}^{\prime}+4
$$

Of course the heterotic model also has additional left-moving field such that the total left-moving central charge has the correct value $c_{L, \text { ws }}=26$.

The right-moving fermions in the heterotic model change the accounting in two ways. First, their central charge contribute $c_{F, \mathrm{ws}}=5$ such that the total right-moving central charge has the correct value $c_{R \text {,ws }}=15$. Second, world-sheet supersymmetry demands that these fermions are organized into $\widehat{S L}(2) \times \widehat{S U}(2) \times \widehat{U}(1)^{4}$ current algebra. They contribute $k_{F}=-2, k_{F}^{\prime}=2$ such that the total levels on the right side become $k_{R \text {,tot }}=k_{B}-2$, $k_{R, \text { tot }}^{\prime}=k_{B}^{\prime}-2$.

In order to fully specify the model we must find $k_{B}$, which then determined the level of all the world-sheet current algebras. String theory on $\mathrm{AdS}_{3}$ 42, 43 has a spacetime Virasoro algebra with central charge related to the level of the world-sheet $\widehat{S L(2)}$ current as

$$
c_{\text {spacetime }}=6 k_{\text {tot }} N \text {. }
$$

The overall factor of $N$ is due to winding of the map between string world-sheet and spacetime target. The general construction above describes a bound state of fundamental strings together with NS fivebranes. We now try to get rid of the fivebranes. Given only fundamental strings, our expectation from spacetime considerations is $c_{R}=12 N$, $c_{L}=24 N$ (with $1 / N$ corrections coming from string loop corrections). Then (7.3) indicates $k_{B}=k_{L \text {,tot }}=4$ by considering the left-movers. This fixes the right-moving level to $k_{R, \text { tot }}=k_{B}-2=2$ and then (7.3) gives the correct right-moving central charge as well. This result was not automatic so it gives a modest check on the basic accounting.

As we have discussed, the nonlinear superconformal algebras determine the corrections of (7.3) due to string loops. In principle such corrections could be verified by direct computation of string loops in the $\sigma$-model. Alhough it is unlikely that such explicit computations are ever going to be practical it is meaningful that there is a concrete and nontrivial prediction.

We next consider the $S U(2)$ factor of the world-sheet theory. According to (7.1) with $k_{B}=4$ the bosonic $S U(2)$ level becomes $k_{B}^{\prime}=0$. All that remains is then $k_{R}^{\prime}=2$ from 
the right-moving fermions. The symmetry is therefore reduced, from $S U(2)_{R} \times S U(2)_{L}$ in a generic $\sigma$-model to $S U(2)_{R}$. This is consistent with describing a spacetime $S^{2}$ rather than $S^{3}$. In the present context this is just what we want. The disappearance of one of the bosonic $S U(2)$ 's is reminiscent of the model for fundamental strings presented in [44].

According to 42,43] a world-sheet current algebra at level $k_{\mathrm{ws}}$ gives rise in spacetime to an affine current with the level

$$
k_{\text {spacetime }}=k_{\mathrm{ws}} N \text {. }
$$

In the present case, the world-sheet $\widehat{S U}(2)$ with level $k_{R}^{\prime}=2$ gives a spacetime $\widehat{S U}(2)$ with level $2 N$, in agreement with (3.12). It is also worth noting that $\widehat{S U}(2)_{2}$ can be bosonized to a supersymmetric to a supersymmetric $\widehat{U}(1)$ with the boson at the self-dual radius. This is the value (2.8) of the fifth circle that appears in the classical geometry.

As an aside, we make the following suggestive observation. Since all the right-moving world-sheet fermions are free they form an $\widehat{S O}(8)$ at level 1 , as is familiar from strings in flat space. According to (7.4) this would give a spacetime $\widehat{S O}(8)$ at level $k_{S O}(8)=N$. Since we would like to compactify five of the bosonic directions (although our construction only has a manifest $\left.T^{4}\right)$ the spectrum generically respects only the $\widehat{S O}(3)_{1} \subset \widehat{S O}(8)_{1}$ subgroup, which appeared above as an $\widehat{S U}(2)$. At some points in moduli space the spectrum respects the centralizer as well, an $\widehat{S O}(5)$ at level 1. According to (7.4) this gives a spacetime $\widehat{S O}(5)$ at level $N$.

The direct construction of the superisometry was given in [28]. The corresponding nonlinear algebra is $\widehat{O S p}\left(4^{*} \mid 4\right)$. This algebra has spacetime R-symmetry $\widehat{S O}(3)_{-2 N} \times$ $\widehat{S O}(5)_{N}$. It is not clear what one is to make of the negative level, and the related fact that the representations appearing in the spacetime spectrum are nonunitary. It is therefore also unclear whether one should take seriously the apparent match between (the absolute value of) these levels and those discussed in the previous paragraph.

So far we focussed on the bosonic symmetries and found promising results using simple and rather robust arguments. We next consider supersymmetry which will turn out to be more confusing. The world-sheet theory must of course respect the right-moving supersymmetry because it is gauged. Having introduced a bosonic $\widehat{S L}(2)_{4}$ the supercurrent must be appropriate for $\widehat{S L}(2)$ as well. In the present context we can take

$$
T_{F}=\eta_{A B} \psi^{A} j^{B}-\frac{i}{6} \epsilon_{A B C} \psi^{A} \psi^{B} \psi^{C}-\frac{i}{6} \epsilon_{A^{\prime} B^{\prime} C^{\prime}} \chi^{A^{\prime}} \chi^{B^{\prime}} \chi^{C^{\prime}}+\lambda^{i} \partial Y_{i},
$$

where $j^{B}$ denote the bosonic $\widehat{S L}(2)$ currents, $\psi^{A}$ are the $S L(2)$ fermions, $\chi^{A^{\prime}}$ are three fermions forming $\widehat{S U}(2)_{2}$, and $\left(\lambda^{i}, Y_{i}\right)$ realize the supersymmetric $\widehat{U}(1)^{4}$. Bosonizing as usual the 10 fermions into $5 H_{I}$ 's, there are 32 candidate spacetime supersymmetries

$$
Q_{\alpha}=\exp \left(\frac{1}{2} i \sum_{I=1}^{5} \epsilon_{I} H^{I}\right) \text {. }
$$


Mutual locality imposes the GSO projection $\prod_{I=1}^{5} \epsilon^{I}=1$ and locality with respect to the world-sheet supercurrent (7.5) further imposes $\prod_{I=1}^{3} \epsilon^{I}=1$. Therefore, there are only 8 spacetime supersymmetries whereas we expected enhancement to 16 supersymmetries. In fact, it is this enhancement that forces the appearance of a the nonlinear superconformal algebra in spacetime, our main interest. The key test for a successful $\sigma$-model is to achieve the correct spacetime supersymmetry.

There is one more important ingredient to consider: the construction may need specific discrete identifications realized by some orbifold. Generally $\sigma$-models realize $S^{2}$ as the Lenz space $S^{3} / Z_{p}$ by taking an asymmetric $Z_{p}$ orbifold of $S U(2)$. The world-sheet central charges discussed above are not affected by such an orbifold, but other symmetries including supersymmetries depend sensitively on such discrete choices. An explicit model in the context of string theory in $\mathrm{AdS}_{3}$ [42,43] was studied in [45]. In this model the currents are invariant under the orbifold at the lowest level $p=2$ and so the model is precisely the one discussed above. This model appears to have only 8 spacetime supersymmetries and so it is not quite the correct dual.

Another approach to the asymmetric orbifold $S^{3} / Z_{p}$ [46] represents some of the leftmovers as fermions and use these to balance the anomaly from the asymmetric gauging of the $S U(2)$ (some useful details are given in [47]). In this model there is an enhanced discrete symmetry which is not manifest when the left-movers are represented as bosons. The special case where the left-moving fermions are neutral under the gauging (so their charges $Q=0$ ) was proposed in [28] as the holographic dual of fundamental strings. However, it is (again) not clear how to achieve the correct spacetime supersymmetry.

\section{Discussion}

In this paper we studied aspects of the holographic description of fundamental heterotic strings, and in particular the hypothesis that they are governed by nonlinear superconformal algebras. There are many open questions and further avenues to pursue; we close by mentioning a few.

There are several arguments supporting the appearance of $\widehat{O S p}\left(4^{*} \mid 4\right)$ in the five dimensional heterotic string. In particular, the identification in [28] of $\operatorname{OSp}\left(4^{*} \mid 4\right)$ as the superisometry group is an excellent clue. It should be possible to actually prove this assertion, at least in the context of five dimensional $R^{2}$ supergravity, by constructing explicitly the generators of the algebra. Note that this is different than finding the superisometry group; indeed, the structure of the full nonlinear algebra implies that the Lie algebra of $\operatorname{OSp}\left(4^{*} \mid 4\right)$ is not in fact a subalgebra of the full $\widehat{O S p}\left(4^{*} \mid 4\right)$, except in the $k \rightarrow \infty$ limit.

Assuming that the nonlinear algebras indeed appear in the present context, to fill in the CFT side of the AdS/CFT correspondence we need to identify boundary CFTs possessing these symmetry algebras. Not much is known about such field theories. 
In [48] Witten gave a proposal for the boundary CFT description of pure gravity in $\mathrm{AdS}_{3}$. It might similarly be worthwhile to consider the CFT dual of pure $\mathrm{AdS}_{3}$ supergravity based on the various supergroups. As we have emphasized here, the Jacobi identities by themselves already lead to highly nontrivial quantum gravity predictions for corrections to the black hole entropy. Pursuing the logic of 48 should lead to further structure.

\section{Acknowledgements:}

We thank A. Dabholkar, J. Davis, E. D'Hoker, D. Marolf, A. Sen, A. Strominger and E. Witten for discussions. FL thanks CERN for hospitality as the write-up was completed. The work of PK is supported in part by NSF grant PHY-0456200. The work of FL is supported by DOE under grant DE-FG02-95ER40899. The work of AS is supported by an NSF IGERT Fellowship.

\section{Appendix A. Conventions and normalizations}

The detailed expressions for the nonlinear superconformal algebras depend on many group theory conventions, which can differ among the cited references [8-14]. For convenience, here we give our conventions and explain the relation of our formulas to those in the references. Our primary reference is [19].

\section{A.1. Basic Lie algebra}

The scalar product of two Lie algebra elements $X$ and $Y$ is given by the basis independent Killing form

$$
K(X, Y)=\frac{1}{2 g} \operatorname{Tr}(a d X a d Y),
$$

where $g$ is the dual Coxeter number, defined below. We normalize the generators of the Lie algebra such that $K\left(T^{a}, T^{b}\right)=\delta^{a b}$. Roots are introduced by writing the algebra in its standard Cartan form

$$
\left[H^{i}, E^{\alpha}\right]=\alpha^{i} E^{\alpha},
$$

with $H^{i}$ properly normalized. We take the long roots of the algebra to have length squared $\psi^{2}=2$.

All other normalizations are fixed by these conventions. The generators in some general representation $\rho$ are normalized according to

$$
\operatorname{Tr}_{\rho}\left(T^{a} T^{b}\right)=2 x_{\rho} \delta^{a b},
$$

which defines the Dynkin index $x_{\rho}$. The dual Coxeter number is

$$
g=x_{\mathrm{adj}}=\frac{1}{2} C_{2}(\operatorname{adj}),
$$


where the quadratic Casimir in the adjoint representation is defined by

$$
f^{a c d} f^{b c d}=C_{2}(\operatorname{adj}) \delta^{a b}
$$

In these conventions we have:

\begin{tabular}{|c|c|c|}
\hline Algebra & Dual Coxeter $g$ & Dynkin index of defining rep. \\
\hline$S U(n)$ & $\mathrm{n}$ & $\frac{1}{2}$ \\
\hline$S O(n>3)$ & $\mathrm{n}-2$ & 1 \\
\hline$S p(2 n)$ & $\mathrm{n}+1$ & $\frac{1}{2}$ \\
\hline
\end{tabular}

Table 3: basic normalizations of some important Lie algebras

In the important case of $S O(3) \simeq S U(2)$ we cannot use $n=3$ in $S O(n)$ because precisely for this case the long root disappears, and so the normalization is off. To compute the Dynkin index of the vector representation of $S O(3)$ we consider instead the adjoint of $S U(2)$ and so get $x_{v}(S O(3))=x_{\mathrm{adj}}(S U(2))=g(S U(2))=2$.

\section{A.2. Conventions for $W Z W$ models}

We follow [49] and write the topological term in the WZW model as

$$
k \Gamma=\frac{-i k}{24 \pi x_{\rho}} \int \operatorname{Tr}_{\rho}\left[\left(g^{-1} d g\right)^{3}\right]
$$

where $\operatorname{Tr}_{\rho}$ represents the trace in the representation $\rho$ and the Dynkin index was introduced in (A.3). The factor of $x_{\rho}$ in the denominator of (A.6) makes $k \Gamma$ independent of the choice of $\rho$. According to [49] the WZW model with our normalizations has the current algebra OPE

$$
J^{a}(z) J^{b}(0) \sim \frac{k \delta^{a b}}{z^{2}}+i f^{a b c} \frac{J^{c}(0)}{z},
$$

which is equivalent to the algebra

$$
\left[J_{n}^{a}, J_{m}^{b}\right]=i f^{a b c} J_{n+m}^{c}+k n \delta^{a b} \delta_{n+m}
$$

For the action to be well defined the level $k$ must be an integer for any group; for $S O(3)$ it must be an even integer. When comparing (A.7), (A.8) with the literature it is essential to normalize the currents consistent with (A.4), (A.5), i.e. such that $f^{a d e} f^{b d e}=C_{2}(\operatorname{adj}) \delta^{a b}=$ $2 g \delta^{a b}$. 


\section{A.3. Central charges of nonlinear algebras}

In order to read off the correct results for the central charges of the nonlinear SCAs we must apply the normalizations above carefully. Some examples:

$\widehat{O S p}(n \mid 2: R)$

Knizhnik [8] uses $f^{a b c} f^{a b d}=2(n-2) \delta^{c d}=2 g_{S O(n)} \delta^{c d}$ and writes the OPE as (A.7), albeit with $k \rightarrow S$. This agrees with our normalizations so $S_{\text {there }}=k_{\text {here }}$ and then $\mathbb{8}$ gives

$$
c=\frac{k\left(6 k+n^{2}-10\right)}{2(k+n-3)}=3 k+\ldots .
$$

$\widehat{S U}(1,1 \mid n)_{n \neq 2}$

In this case Knizhnik [8] writes $f^{a b c} f^{a b d}=4 N \delta^{c d}=4 g_{S U(N)} \delta^{c d}$ and writes the OPE as (A.7), again with $k \rightarrow S$. Here the normalizations are off so that $S_{\text {there }}=2 k_{\text {here. In }}$ our notation [8] gives

$$
c=\frac{3 k(2 k+n)+(n-1)(1+(n+1) k)}{k+n-1}=6 k+\ldots
$$

$\widehat{F}(4)$

We use [12]. Taking $\psi^{2}=2$ all normalizations agree, so $k_{\text {there }}=k_{\text {here }}$. Then

$$
c=\frac{2 k(2 k+11)}{k+4}=4 k+\ldots
$$

$\widehat{G}(3)$

We use [12]. Taking $\psi^{2}=2$ all normalizations agree again, so $k_{\text {there }}=k_{\text {here }}$. Then

$$
c=\frac{k(9 k+31)}{2(k+3)}=\frac{9 k}{2}+\ldots
$$

$\widehat{S U}(1,1 \mid 2) / U(1)$

This is the standard $\mathcal{N}=4 \mathrm{SCA}$ for which

$$
c=6 k
$$

$\widehat{D^{1}}(2,1 ; \alpha)$ 
This is the "large" $\mathcal{N}=4$ algebra. Our conventions agree with e.g. [50] so that $k_{\text {there }}=k_{\text {here }}$. The result is

$$
c=\frac{6 k_{1} k_{2}}{k_{1}+k_{2}}
$$

$\widehat{O S p}\left(4^{*} \mid 2 m\right)$

We use [12]. We label $\widehat{S U}(2)$ currents as $J^{\alpha \beta}$ and $\widehat{S p}(2 m)$ currents as $J^{A B}$. Both are symmetric (we're using sp convention for $S U(2)$ ). The affine $\widehat{S U}(2)$ algebra is

$$
\left[J_{m}^{\alpha \beta}, J_{n}^{\gamma \delta}\right]=\epsilon^{\beta \gamma} J_{m+n}^{\alpha \delta}+\epsilon^{\alpha \delta} J_{m+n}^{\beta \gamma}+\epsilon^{\alpha \gamma} J_{m+n}^{\beta \delta}+\epsilon^{\beta \delta} J_{m+n}^{\alpha \gamma}-k_{2}\left(\epsilon^{\alpha \gamma} \epsilon^{\beta \delta}+\epsilon^{\beta \gamma} \epsilon^{\alpha \delta}\right) m \delta_{m+n},
$$

and the analogous structure for the $\widehat{S p}(2 m)$ currents with their level being $k_{1}$. Jacobi identities determine the central charge as

$$
c=-\frac{3 k_{2}\left(k_{2}+2 m+4\right)}{k_{2}-2 m+4}+\frac{6 k_{2}+(2 m+1)(m-2)\left(k_{2}+2 m+4\right)}{k_{2}-2 m+4},
$$

and further relate the levels as

$$
2 k_{1}+k_{2}+2 m+4=0 .
$$

For large level the relative signs of $k_{1,2}$ must be opposite. To get a positive central charge we need $k_{2}<0$.

We need to write the $\widehat{S U}(2)$ level $k_{2}$ in our conventions. The commutation relations (A.15) give

$$
\left[J_{0}^{12}, J_{0}^{11} \pm J_{0}^{22}\right]=-2\left(J_{0}^{11} \mp J_{0}^{22}\right),
$$

which identifies $J^{3}=\frac{1}{\sqrt{2}} J^{12}$ as the properly normalized $S U(2)$ Cartan generator which gives roots of length squared 2. Then (A.16) gives

$$
\left[J_{n}^{3}, J_{m}^{3}\right]=\frac{k_{2}}{2} m \delta_{m+n},
$$

and comparison with (A.8) identifies the level in our conventions as $k_{\text {here }}=\frac{1}{2} k_{2}$. 


\section{References}

[1] R. R. Metsaev and A. A. Tseytlin, "Order alpha-prime (Two Loop) Equivalence of the String Equations of Motion and the Sigma Model Weyl Invariance Conditions: Dependence on the Dilaton and the Antisymmetric Tensor," Nucl. Phys. B 293, 385 (1987).

[2] E. A. Bergshoeff and M. de Roo, "The Quartic Effective Action Of The Heterotic String And Supersymmetry," Nucl. Phys. B 328, 439 (1989).

[3] I. Antoniadis, S. Ferrara, R. Minasian and K. S. Narain, "R**4 couplings in M- and type II theories on Calabi-Yau spaces," Nucl. Phys. B 507, 571 (1997) arXiv:hepth/9707013].

[4] K. Hanaki, K. Ohashi and Y. Tachikawa, "Supersymmetric Completion of an $R^{2}$ Term in Five-Dimensional Supergravity," Prog. Theor. Phys. 117, 533 (2007) arXiv:hepth/0611329].

[5] A. Castro, J. L. Davis, P. Kraus and F. Larsen, "5D attractors with higher derivatives," JHEP 0704, 091 (2007) arXiv:hep-th/0702072].

[6] A. Castro, J. L. Davis, P. Kraus and F. Larsen, "5D Black Holes and Strings with Higher Derivatives," arXiv:hep-th/0703087.

[7] J. D. Brown and M. Henneaux, "Central Charges in the Canonical Realization of Asymptotic Symmetries: An Example from Three-Dimensional Gravity," Commun. Math. Phys. 104, 207 (1986).

[8] V. G. Knizhnik, "Superconformal algebras in two dimensions," Theor. Math. Phys. 66, 68 (1986) [Teor. Mat. Fiz. 66, 102 (1986)].

[9] M. A. Bershadsky, "Superconformal algebras in two dimensions with arbitrary N," Phys. Lett. B 174, 285 (1986).

[10] K. Schoutens, "Representation Theory For A Class Of SO(N) Extended Superconformal Operator Algebras," Nucl. Phys. B 314, 519 (1989).

[11] F. Defever, W. Troost and Z. Hasiewicz, "Superconformal algebras with quadratic nonlinearity," Phys. Lett. B 273, 51 (1991).

[12] E. S. Fradkin and V. Y. Linetsky, "Results Of The Classification Of Superconformal Algebras In Two-Dimensions," Phys. Lett. B 282, 352 (1992) [arXiv:hep-th/9203045. "Classification Of Superconformal Algebras With Quadratic Nonlinearity," arXiv:hepth/9207035. "Classification Of Superconformal And Quasisuperconformal Algebras In Two-Dimensions," Phys. Lett. B 291, 71 (1992);

[13] E. S. Fradkin and V. Y. Linetsky, "An Exceptional N=8 superconformal algebra in two-dimensions associated with F(4)," Phys. Lett. B 275, 345 (1992).

[14] P. Bowcock, "Exceptional Superconformal Algebras," Nucl. Phys. B 381, 415 (1992) arXiv:hep-th/9202061. 
[15] B. Bina and M. Gunaydin, "Real forms of non-linear superconformal and quasisuperconformal algebras and their unified realization," Nucl. Phys. B 502, 713 (1997) arXiv:hep-th/9703188.

[16] J. de Boer, "Six-dimensional supergravity on $\mathrm{S}^{* *} 3 \times \mathrm{AdS}(3)$ and $2 \mathrm{~d}$ conformal field theory," Nucl. Phys. B 548, 139 (1999) [arXiv:hep-th/9806104].

[17] K. Ito, "Extended superconformal algebras on AdS(3)," Phys. Lett. B 449, 48 (1999) arXiv:hep-th/9811002.

[18] M. Henneaux, L. Maoz and A. Schwimmer, "Asymptotic dynamics and asymptotic symmetries of three-dimensional extended AdS supergravity," Annals Phys. 282, 31 (2000) arXiv:hep-th/9910013.

[19] J. A. Harvey, R. Minasian and G. W. Moore, "Non-abelian tensor-multiplet anomalies," JHEP 9809, 004 (1998) arXiv:hep-th/9808060.

[20] P. Kraus and F. Larsen, "Microscopic black hole entropy in theories with higher derivatives," JHEP 0509, 034 (2005) arXiv:hep-th/0506176].

[21] P. Kraus and F. Larsen, "Holographic gravitational anomalies," JHEP 0601, 022 (2006) arXiv:hep-th/0508218.

[22] V. Balasubramanian and P. Kraus, "A stress tensor for anti-de Sitter gravity," Commun. Math. Phys. 208, 413 (1999) arXiv:hep-th/9902121.

[23] R. Emparan, C. V. Johnson and R. C. Myers, "Surface terms as counterterms in the AdS/CFT correspondence," Phys. Rev. D 60, 104001 (1999) arXiv:hep-th/9903238.

[24] P. Kraus, F. Larsen and R. Siebelink, "The gravitational action in asymptotically AdS and flat spacetimes," Nucl. Phys. B 563, 259 (1999) [arXiv:hep-th/9906127.

[25] I. Papadimitriou and K. Skenderis, "Thermodynamics of asymptotically locally AdS spacetimes," JHEP 0508, 004 (2005) arXiv:hep-th/0505190].

[26] A. Dabholkar and S. Murthy, "Fundamental Superstrings as Holograms," arXiv:0707.3818 [hep-th].

[27] C. V. Johnson, "Heterotic Coset Models of Microscopic Strings and Black Holes," arXiv:0707.4303 [hep-th].

[28] J. M. Lapan, A. Simons and A. Strominger, "Nearing the Horizon of a Heterotic String," arXiv:0708.0016 [hep-th].

[29] G. Lopes Cardoso, B. de Wit and T. Mohaupt, "Corrections to macroscopic supersymmetric black-hole entropy," Phys. Lett. B 451, 309 (1999) arXiv:hep-th/9812082.

[30] A. Dabholkar, "Exact counting of black hole microstates," Phys. Rev. Lett. 94, 241301 (2005) arXiv:hep-th/0409148.

[31] A. Sen, "How does a fundamental string stretch its horizon?," JHEP 0505, 059 (2005) arXiv:hep-th/0411255.

[32] V. Hubeny, A. Maloney and M. Rangamani, "String-corrected black holes," JHEP 0505, 035 (2005) arXiv:hep-th/0411272. 
[33] A. Sen, "Stretching the horizon of a higher dimensional small black hole," JHEP 0507, 073 (2005) arXiv:hep-th/0505122.

[34] J. Hansen and P. Kraus, "Generating charge from diffeomorphisms," JHEP 0612, 009 (2006) arXiv:hep-th/0606230.

[35] W. Nahm, "Supersymmetries and their representations," Nucl. Phys. B 135, 149 (1978).

[36] P. Kraus and F. Larsen, "Partition functions and elliptic genera from supergravity," JHEP 0701, 002 (2007) arXiv:hep-th/0607138.

[37] E. Witten, "Quantum field theory and the Jones polynomial," Commun. Math. Phys. 121, 351 (1989).

[38] G. W. Moore and N. Seiberg, "Taming the Conformal Zoo," Phys. Lett. B 220, 422 (1989).

[39] S. Elitzur, G. W. Moore, A. Schwimmer and N. Seiberg, "Remarks On The Canonical Quantization Of The Chern-Simons-Witten Theory," Nucl. Phys. B 326, 108 (1989).

[40] S. Carlip, "Conformal field theory, (2+1)-dimensional gravity, and the BTZ black hole," Class. Quant. Grav. 22, R85 (2005) arXiv:gr-qc/0503022.

[41] T. Regge and C. Teitelboim, "Role Of Surface Integrals In The Hamiltonian Formulation Of General Relativity," Annals Phys. 88, 286 (1974).

[42] A. Giveon, D. Kutasov and N. Seiberg, "Comments on string theory on AdS(3)," Adv. Theor. Math. Phys. 2, 733 (1998) arXiv:hep-th/9806194.

[43] D. Kutasov and N. Seiberg, "More comments on string theory on AdS(3)," JHEP 9904, 008 (1999) arXiv:hep-th/9903219.

[44] A. Giveon and D. Kutasov, "Fundamental strings and black holes," JHEP 0701, 071 (2007) arXiv:hep-th/0611062.

[45] D. Kutasov, F. Larsen and R. G. Leigh, "String theory in magnetic monopole backgrounds," Nucl. Phys. B 550, 183 (1999) arXiv:hep-th/9812027.

[46] S. B. Giddings, J. Polchinski and A. Strominger, "Four-dimensional black holes in string theory," Phys. Rev. D 48, 5784 (1993) arXiv:hep-th/9305083.

[47] C. V. Johnson, "Exact models of extremal dyonic 4-D black hole solutions of heterotic string theory," Phys. Rev. D 50, 4032 (1994) arXiv:hep-th/9403192.

[48] E. Witten, "Three-Dimensional Gravity Revisited," arXiv:0706.3359 [hep-th].

[49] P. Di Francesco, P. Mathieu and D. Senechal, "Conformal Field Theory," New York, USA: Springer (1997) $890 p$

[50] S. Gukov, E. Martinec, G. W. Moore and A. Strominger, "An index for 2D field theories with large $\mathrm{N}=4$ superconformal symmetry," arXiv:hep-th/0404023. 